\title{
The quick carotid scan for prevention of strokes due to carotid artery disease
}

\author{
George S. Lavenson Jr, Charles A. Andersen
}

${ }^{1}$ Department of Surgery, Uniformed Services University, Bethesda, MD, USA; ${ }^{2}$ Department of Surgery, Madigan Army Medical Center, Tacoma, WA, USA

Contributions: (I) Conception and design: Both authors; (II) Administrative support: Both authors; (III) Provision of study materials or patients: Both authors; (IV) Collection and assembly of data: Both authors; (V) Data analysis and interpretation: GS Lavenson; (VI) Manuscript writing: All authors. (VII) Final approval of manuscript: Both authors.

Correspondence to: George S. Lavenson Jr, MD, FACS, RVT. Adjunct Professor of Surgery, Uniformed Services University, 42 Ala Moana Street, Lahaina, HI 96761, USA. Email: glavenson@aol.com.

\begin{abstract}
There are approximately 800,000 strokes in the United States (U.S.) annually. This number has remained the same for decades despite efforts at prevention. The Center for Disease Control (CDC) estimates that $80 \%$ of strokes could be prevented. A prime reason for failure of prevention is that the three immediate modifiable causes of strokes, carotid artery disease (CAD), atrial fibrillation (AFib), and hypertension (HTN) are asymptomatic in $80 \%$ of cases prior to the stroke. Strokes occur predominantly in seniors and the only possible means of reducing strokes on a large scale is to screen seniors for the asymptomatic disease so that it can be preemptively managed. We present a quick, accurate and cost-effective method of screening the senior population for asymptomatic carotid disease. The technique is a quick carotid scan (QCS). The QCS is a 1-minute long, image only, rapid, color flow ultrasound scan of the cervical carotid arteries that had a sensitivity of $97 \%$ when evaluated at New York University (NYU). Once identified by the QCS the approximately 8\% of those screened found to have a positive QCS can then be referred for a full carotid duplex ultrasound (DUS). Those patients with a positive DUS can then be referred for further evaluation and appropriate stroke prevention management. The use of a full carotid DUS for screening widely for carotid disease is too time consuming and too costly. Approximately 160,000 or nearly $20 \%$ of the 800,000 strokes that occur annually in the U.S. are due to CAD that could in large part be prevented by screening the senior population with the QCS, finding those with CAD, evaluating them, and preemptively managing them prior to the occurrence of the stroke
\end{abstract}

Keywords: Stroke; prevention; screen; carotid arteries

Submitted Nov 26, 2020. Accepted for publication Mar 18, 2021.

doi: $10.21037 /$ atm-20-7687

View this article at: http://dx.doi.org/10.21037/atm-20-7687

There have been approximately 800,000 strokes annually in the United States (U.S.) for decades without reduction according to the Center for Disease Control (CDC) $(1,2)$. The CDC also estimates that if unchecked, there will be over 1 million strokes annually by 2023 , but the CDC estimates that over $80 \%$ of those strokes could be prevented (2).

Prevention of strokes on a large scale is possible because the immediate causes of strokes, carotid artery disease
(CAD), atrial fibrillation (AFib), and hypertension (HTN), are asymptomatic in $80 \%$ of cases prior to the strokes they cause, but if found, evaluated, and managed preemptively, strokes could be prevented on a large scale $(3,4)$

The key fact that allows prevention of strokes from $\mathrm{CAD}$ is that the CAD that cause strokes is asymptomatic in $80 \%$ of cases prior to the strokes it causes (2). Finding the silent, asymptomatic carotid stenosis (ACS) in seniors who have the bulk of the strokes, so that it can be managed 
preemptively provides the opportunity to prevent the majority of strokes from CAD. Although many have recommended against screening for ACS including the United States Preventive Services Task Force (USPSTF) $(5,6)$, a large number of strokes could be prevented and large attendant expenses reduced by this means (7-12).

While finding the ACS could be attempted in a number of ways, little known quick carotid scan (QCS) (12) seems the best means in that its speed and accuracy makes it most inclusive.

\section{Method}

The QCS was envisioned in the late 1990's when author LG noted that on scanning the carotid arteries of patients in the office for a standard duplex ultrasound (DUS) examination, it was immediately apparent on the initial image only scan with the probe whether the carotid artery was normal or had disease. Based on this observation, it occurred that this could be used to screen seniors and ferret out the average of $8 \%$ of seniors with an indication of CAD so that the longer, more complicated and costly DUS examination could be employed on those found to have a positive screen. This initial image only scan was termed a QCS. A positive scan was indicated by the presence of a visible lesion, color shift caused by increased flow velocity signaling stenosis even in a non-visible sonolucent lesion, or an indeterminate scan (12). All were referred on for a full DUS.

A protocol for screening for CAD was then developed and was employed in pilot screening events for seniors at Kaweah Delta District Hospital (KDDH) in Visalia, CA, and Madigan Army Medical Center (MAMC) in Tacoma, WA. Additional experience and further development of the QCS protocol was accomplished with 76 screening event at 26 hospitals in the U.S. and Canada (13-19).

The QCS then is an image only rapid scan of the carotid artery that can be done in one minute or less. The screening protocol does not include time consuming patient histories, the complexity of velocity measurements, hard copies, or counseling as would be done with a conventional full DUS examination. We found that a wide breath of health care professionals at KDDH, MAMC, and other medical facilities could do the simple, image only carotid scan with little instruction. The goal of the QCS is to rapidly scan the cervical carotid artery of the senior population to find and select out what was an average of $8 \%$ of seniors. who had a visible carotid lesion, color shift, or an indeterminate scan.
These were sorted out for a later more lengthy full DUS, further evaluation, and appropriate management. The QCS is extremely accurate and had a sensitivity of $97 \%$ and specificity of $93 \%$ when evaluated at New York University (NYU) (20).

Seniors could obtain a QCS either by a physician referral or self-referral to a QCS examination site, and thus could obtain the QCS even without agreement from the general medical hierarchy, a personal care giver, or the government-such as one can do in obtaining a flu shot. The senior can also simply appear, line up as for a flu shot, and sit on a chair or stool during the scan. On learning of the potential stroke risk of CAD, the senior population has had high interest in getting stroke prevention carotid examinations when available. Over 8 million of the 48 million seniors in the U.S. have paid a commercial organization to have an even more prolonged and costly conventional DUS carotid examination.

Seniors and their families can be advised of the rationale of carotid screening for stroke prevention, and the availability of the screening sites by notices in newspapers, posters, pamphlets, and on television, much as is done by the commercial organization.

Screening sites for the QCS can be established in medical facilities or a diversity of sites locally or regionally. Seniors can be scheduled or taken in order at the sites and either lie on an examination table or simply sit on a stool or chair during the rapid scanning. The QCS can be done as quickly, commonly, and easily for the senior population as obtaining a flu shot or a Covid screen and with less discomfort. It is done even more rapidly and easily than a Pap Smear, or colonoscopy etc. The skill to accomplish the QCS is easily transferable. With brief instruction at MAMC, health care givers at any level or specialty were able to do the QCS quickly and accurately. At the conclusion of the scan, the senior is simply advised whether the scan is normal, has minimal disease for which it is advised that the scan be repeated in 1 year, or is positive in which case arrangements are made for a follow-up full DUS examination in a certified vascular laboratory with indicated further evaluation and management following.

Unlike the commercial organization, screening in the local medical facilities and QCS sites can be offered to seniors at little or no cost. While Medicare in the U.S. does not now cover the cost of carotid screening, it does reimburse for the further evaluation and management of disease found on screening, which can cover the nominal monetary outlay of a medical facility to provide the initial 
screening.

Follow up of those seniors found to have ACS can be done by the local medical facility where the screening was done, arranged by the primary caregiver, or accomplished by referral to a specialist or specialty facility for the condition found.

With further development of small, hand-held screening devices the size of an iphone that can already show a visible lesion and color flow images, the addition of pulsed Doppler capability could show the color shift due to increased flow velocity that accompanies the presence of stenotic lesions and thus could also allow identification of those non-visible sono-lucent but stenotic lesions. This would expand use of the QCS to more sites and a QCS could be done as part of an in-hospital evaluation of a patient in a room or ward, on teaching rounds in hospitals, or in a doctor's office as part of an annual or diagnostic examination-this in lieu of listening for an audible bruit with a stethoscope which has a sensitivity of only $50 \%$ (1).

\section{Results}

The results from screening the senior population for ACS with the QCS has provided both important clinical information in its own right, and data needed to estimate the number of strokes that could be prevented and the accompanying expenditures possibly reduced. The data herein allows estimation of the accuracy of the QCS, the incidence of ACS in the senior population, the risk of stroke from the ACS, and an estimation of the number of strokes and the amount of expenditure that could be prevented by broadly screening the senior population for ACS with the QCS. The rapidity of the QCS and ability to screen unlimited numbers of the senior population can provide uniform, accurate, and very large accumulations of data without the variability of many methods and studies. The near total proportion of the senior population capable of being screened provides confidence of the data beyond other studies.

The accuracy of the QCS was first determined in the office of author LG, who is both a vascular surgeon and a registered vascular technologist, by performing a QCS, following it with a full DUS, and comparing the results on many patients. The sensitivity and specificity of the QCS were $93 \% / 87 \%$ respectively. When evaluated at NYU with a different technologist performing the QCS and the following full DUS, the sensitivity and specificity of the QCS were 97\%/93\% respectively.
The incidence of CAD in seniors was determined in 2002-2003 by staff screening of 22,496 seniors in 75 institutions in the U.S. and Canada including those by the Society for Vascular Surgery (SVS), the Society for Vascular Ultrasound (SVU), the American Vascular Association (AVA), NYU, MAMC, and KDDH in Central California. The resulting incidence of CAD rounded off to a rather uniform $8 \%(20)$. The principal investigators of the study were author LG and Bonnie Johnson, the then Chief Vascular Technologist of the Vascular Laboratory at Stanford University. The statistics were done at Stanford University.

A side compilation of the results found that the sensitivity of even combinations of the most common and related risk factors (RFs) included in the Framingham Risk Scale ranged only from $60 \%$ to $70 \%$.

The 5-year risk of stroke from ACS without discovery or management has been estimated to be $15-20 \%$. The risk of stroke would of course be significantly higher than the $10 \% / 11 \%$ incidence of stroke with management as in the Asymptomatic Carotid Arteriosclerotic Study (21) and the asymptomatic carotid surgical trial (22). Both carotid endarterectomy (CEA) and the improved "best medical treatment" (BMT), can now lower the 5-year risks of stroke to well less than $3 \%$, an even further difference from the stroke risk of 15-20\% with unknown and unmanaged ACS. The improvement in the stroke risk with BMT from the earlier $10 \%$ risk levels at 5 years does not detract, as some would argue, from the need to find the unknown ACS so that it can be treated, either medically or by intervention, but in fact enhances that need.

The number of strokes caused annually by ACS can be estimated from the above results and additional data. Since $8 \%$ of seniors have ACS, of the 48 million seniors in the U.S., 3,480,000 would have ACS. With a $20 \%$ risk of stroke from ACS in 5 years with no discovery or management of the $3,480,000$ seniors with ACS, 768,000 would be anticipated to have a stroke in 5 years or 153,600 in 1 year. This is $19 \%$ of the annual stroke incidence of 800,000 (2). These figures may well be conservative since the incidence of ACS in seniors in the study above was primarily determined in urban areas but was found to be as high as $25 \%$ in smaller rural communities in the stroke belt of Central California.

The monetary savings from strokes that could be prevented by screening seniors with the QCS can also be estimated. A cost of $\$ 182,000$ per stroke was estimated by a committee of the SVS and AVA, a per stroke cost of $\$ 124,000$ 
was reported from Dartmouth, and the median figure of the two was $\$ 147,000$ which was similar to the stroke costs in Central California. On subtracting the cost of screening sufficient seniors to prevent a single stroke, performing a DUS on those seniors found to have CAD on screening, further evaluation costs as necessary, and the cost of the CEA's required to prevent 1 stroke totaled $\$ 83,000$; thus, the savings per stroke prevented was $\$ 64,000$ (17).

With a near negative peri-operative stroke risk of $<1 \%$ in three all-inclusive and consecutive, CEA series of over 60,000 patients in Maryland, New England, and California (17), and low stroke risk results with BMT, the potential for reducing direct Medicare stroke expenditures due to ACS exceeds 50 billion dollars annually. With inclusion of indirect stroke costs, the savings in expenditures has been estimated to exceed 200-300 billion dollars/year. Although these studies may be dated and there has been some inflation, the relative relationships most likely remain valid and stroke persists as the leading long-term lineitem expenditure for Medicare. By screening the senior population with the QCS, this expenditure could be greatly reduced.

The QCS was used in multiple stroke prevention screening events in the Central Valley of California "stroke belt", and has been employed as part of an annual medical evaluation for military retirees at MAMC for many years with discovery of significant CAD. The QCS has been used at NYU for years as a means of dealing with the large number of carotid examinations requested.

\section{Discussion}

\section{General}

Unlike the other major medical conditions of heart attacks, cancer, pulmonary disease, and trauma, there is a potential opportunity to prevent and reduce strokes on a truly large and epidemiological scale-a potential breakthrough and minimization of a major medical condition.

Preventing strokes on an epidemiological scale is possible because the three immediate causes of stroke are generally asymptomatic prior to the strokes they cause, but are discoverable by screening, and can be successfully treated once found.

A dominant reason why strokes due to ACS have not been prevented sufficiently to reduce the total number of strokes in large measure, is that much of the focus has been on how to treat the ACS, but not on how to find the ACS in the first place so that it can be managed.

But by focusing on screening seniors to find the silent ACS so that it can be treated, there is the potential of reducing the 5 -year risk of stroke from CAD of $15-20 \%$ with no discovery or treatment, to less than $3 \%$ with either interventional or medical management.

The approaches to the silent ACS varies from not screening for the ACS to different means of finding the ACS.

\section{Not screening}

A recommendation against screening the "general adult population" for ACS was made by the USPSTF first in 2007 (5) and has been recently updated. Each justification below given by the USPSTF for the recommendation against screening for ACS is followed by the authors reasons why those justifications against screening are not valid (23).

(I) USPSTF: the incidence of CAD in the general population is only $1 \%$, making screening not of value or cost-effective. But strokes occur almost entirely in the senior population where the incidence of CAD is not $1 \%$ but rather $8 \%$ (20). With a $1 \%$ incidence of CAD in the general adult population it would be necessary to screen nearly 2,500 in that general population to prevent one stroke; here as with an $8 \%$ incidence of CAD in the senior population screening only 300 seniors is required to prevent a stroke. The 153,000 strokes that are potentially preventable from seniors annually are $19.2 \%$ of the annual number of strokes of 800,000 .

(II) USPSTF: few strokes are caused by CAD. Not true: nearly 160,000 or $20 \%$ of the 800,000 strokes annually are caused by CAD, as shown above.

(III) USPSTF: screening leads to a conventional DUS which is not accurate. The DUS is accurate, particularly if performed by a registered vascular technologist and in a certified vascular laboratory. When the CEA is performed under local anesthesia which allows full visualization of the lesion prior to incising or altering it, the lesion in the experience of author LG is usually more severe than the preoperative tests would indicate. In addition, the results of the DUS can be followed by additional confirmatory tests as needed (24).

(IV) USPSTF: angiography for further evaluation is dangerous. Angiography is seldom used now for 
this purpose and if additional confirmation or evaluation is needed beyond the DUS it is generally accomplished by an MRI or CTA (24).

(V) USPSTF: screening can result in a CEA which is dangerous. Consecutive and all-inclusive series of over 60,000 CEA's with peri-operative stroke rates of $1 \%$ or less have been reported from Maryland, California, and New England (25). The possibility of treatment with a CEA is not a valid reason to avoid screening and not finding the ACS that can lead to a 5 -year stroke in $15-20 \%$ of cases if not found and managed and the stroke risk lowered to less than $3 \%$ with a CEA.

In a presentation to the SVU in 2008 and a publication in 2012 in the Journal of Vascular Ultrasound (23) author LG recommended that these positions of the USPSTF against screening for ACS should be reconsidered for the reasons above, but their position and the number of strokes annually has persisted unchanged.

\section{Use of a cervical bruit to select for a full DUS}

It has long been acknowledged that a cervical bruit can be an indication of possible underlying ACS. Most physicians will auscultate over the cervical carotid artery with a stethoscope as part of a senior's annual physical examination and will refer the patient with an audible bruit for a carotid DUS. But the sensitivity of an audible bruit is only $50 \%$ or less (20) and over half of those with significant ACS will not have an audible bruit. In the same time a QCS could be used with a sensitivity of $97 \%$ compared to listening for a bruit with a sensitivity of only $50 \%$.

\section{Use of RFs to select for a full DUS}

It occurred to many clinicians that the patients they were seeing with cardio-vascular disease and associated causative factors should be checked for CAD (26-28). Thus, the presence of standard RFs has been used by some to select for a full carotid DUS examination and is currently being considered for inclusion in the current guidelines for obtaining a DUS by a large combine of medical organizations in the Extracranial Carotid and Vertebral Artery Disease Guideline and Practice Guidelines of the SVS.

While use of RFs to select those seniors with a higher probability of having ACS as an indication for a full DUS carotid artery examination is a valid option, and a very much needed good step forward from not screening, there are factors that can compromise its full usefulness in discovery of a majority of ACS and wide stroke prevention. A major drawback in using solely RF's to select seniors for a full DUS is that the sensitivity of even combinations of the most sensitive RF's is only $60-70 \%$ (20); thus, $30-40 \%$ with ACS are missed by that means of selection and a significant number who have stroke potential ACS can go unrecognized and unmanaged. The fact is that any senior can have an asymptomatic, silent, stroke potential carotid lesion, and carotid arteries in seniors need to be screened widely to truly reduce strokes on an epidemiological scale.

Additional problems with using the presence of RF's as the dominant and sole means of selecting for a full carotid DUS examination includes the need for agreeable and involved physicians to initiate and go through the process, multiple office visits, laboratory tests, significant costs the length of time required for the above, and the additional 30-45 minutes for a full or even modified DUS as recommended by the Intersocietal Accreditation Commission for those selected for a "screening" DUS.

Using RF's to select for a DUS does find ACS and is of value, but it cannot evaluate the sheer numbers of seniors necessary to find the amount of ACS present in the senior population that would be needed to reduce the number of strokes due to CAD on a large or epidemiological scale. Nonetheless, use of RF's is far better than not screening, and could open the door to further augmentation by the QCS in the future.

\section{Use of a guideline for the management of patients with extracranial carotid and vertebral artery disease (ECVD)}

This is a guideline developed and published in 2011 by a group of multiple vascular societies with new, in process, on-going updates. As part of the guideline, it was recommended that seniors who have a cervical bruit, or two or more standard RFs such as a family history of stroke, HTN, or smoking, or do have a "suspicion of having CAD", be screened for ACS even though there had been no cerebrovascular events or symptoms of CAD. Those with indications for screening would then under-go a complete and full DUS carotid examination in a certified vascular laboratory by a registered vascular technologist.

This is similar to the guideline being considered by the SVS and is a good step away from the USPSTF Guideline of not screening asymptomatic seniors for ACS as mentioned above, and it could potentially result in a 
significant reduction in strokes. But the approach and the short comings of low sensitivity, lack of complete inclusivity, and long examination times required are similar to that of the RF approach, and would fail to have the capability of screening the majority of the senior population that is necessary to reduce strokes more comprehensively. But like use of RFs now gaining some acceptance, this could also be augmented by the QCS.

\section{Use of the QCS to select those in the senior population for a full DUS}

A QCS with non-invasive color flow ultrasound imaging and pulsed Doppler can be done in one minute or less and highly accurately with a sensitivity of $97 \%$. Hence, the QCS has the speed, accuracy, inclusivity, and capability of screening virtually the entire senior population for ACS. The $8 \%$ of seniors with CAD can then be ferreted out and the full DUS examinations focused on those with CAD with further evaluation, and appropriate management following. The three simple steps to prevent strokes from CAD are \#1 screen the senior population with the QCS to find the CAD, \#2 evaluate those positive on screening with a DUS, and \#3 manage the CAD appropriately for the patient (29).

Since the concept of the QCS to screen the senior population for silent CAD and affect prevention of a major medical condition is a different concept than that commonly held, it has been difficult to obtain consideration of large-scale stroke prevention screening by this means. As noted by author CA, it may not be recognized by many that this recommendation for carotid screening with the QCS is not screening with a time consuming standard DUS, but rather it is screening with a new 1 minute highly accurate ultrasound scan to select out the $8 \%$ of seniors with some CAD for further evaluation with the DUS. This is not conjecture-this screening method was used operationally and successfully for a long period for annual screening of retired military personnel by author CA at MAMC (30).

\section{Conclusions}

Stroke is a major and devastating medical condition that preferentially occurs in seniors, fills one-half of nursing homes, is the leading long-term line-item Medicare expenditure, and has persisted without reduction for decades. According to the CDC, $80 \%$ of strokes could be prevented.
Significant CAD which is one of the three immediate causes of stroke is asymptomatic in $80 \%$ of cases prior to the stroke it causes, but if this cause of stroke can be found by screening the senior population with the QCS and managed preemptively, most of those strokes could be prevented.

The only means at the present of reducing strokes from $\mathrm{CAD}$ on a large scale is by screening the senior population with the QCS to find the $8 \%$ with ACS which can be done in less than I minute and with a sensitivity of $97 \%$ to be followed in those positive with a DUS and appropriate stroke preventing management. In addition to the QCS, there is the option of including an ultrasound scan of the abdominal aorta to discover aortic aneurysms prior to rupture and of scanning the common femoral artery to identify arteriosclerotic plaques which have a high correlation with coronary artery disease and can be a trigger for a cardiac and metabolic evaluation $(31,32)$.

\section{Acknowledgments}

Funding: This work was supported by the SVS, SVU, AVA, $\mathrm{KDDH}$, and MAMC.

\section{Footnote}

Provenance and Peer Review: This article was commissioned by the Guest Editor (Kosmas I. Paraskevas) for the series "Carotid Artery Stenosis and Stroke - Prevention and Treatment Part II" published in Annals of Translational Medicine. The article has undergone external peer review.

Peer Review File: Available at http://dx.doi.org/10.21037/ atm-20-7687

Conflicts of Interest: Both authors have completed the ICMJE uniform disclosure form (available at http://dx.doi. org/10.21037/atm-20-7687). The series "Carotid Artery Stenosis and Stroke - Prevention and Treatment Part II" was commissioned by the editorial office without any funding or sponsorship. The authors have no other conflicts of interest to declare.

Ethical Statement: The authors are accountable for all aspects of the work in ensuring that questions related to the accuracy or integrity of any part of the work are appropriately investigated and resolved.

Open Access Statement: This is an Open Access article 
distributed in accordance with the Creative Commons Attribution-NonCommercial-NoDerivs 4.0 International License (CC BY-NC-ND 4.0), which permits the noncommercial replication and distribution of the article with the strict proviso that no changes or edits are made and the original work is properly cited (including links to both the formal publication through the relevant DOI and the license). See: https://creativecommons.org/licenses/by-nc-nd/4.0/.

\section{References}

1. Lavenson GS Jr. Ultrasound of the carotid arteries. A review and perspective. Vasc Ultrasound Today 1996;1:310.

2. Centers for Disease Control and Prevention. Underlying Cause of death, 1999-2018. CDC WONDER Online Database. Atlanta: Centers for Disease Control and Prevention, 2018. Accessed March 12, 2020.

3. Barnes RW. Gentle on my mind: the epidemiology of stroke. J Vasc Tech 1993;22:37-41.

4. Lavenson GS Jr. Noninvasive vascular testing--a 35-year reflection. World J Surg 2005;29 Suppl 1:S64-6.

5. U.S. Preventive Services Task Force. Screening for carotid artery stenosis: U.S. Preventive Services Task Force recommendation statement. Ann Intern Med 2007;147:854-9. Erratum in: Ann Intern Med 2008;148:248.

6. Perry JR, Szalai JP, Norris JW. Consensus against both endarterectomy and routine screening for asymptomatic carotid artery stenosis. Arch Neurol 1997;54:25-8.

7. Lavenson GS Jr, Sharma D. Cost savings of carotid endarterectomy: value of one vascular surgeon in one year. Perspec Vasc Surg 1994;7:1-15.

8. Lavenson GS Jr, Sharma D. Medical cost savings through stroke prevention from 100 consecutive new carotid duplex scans. Cardiovasc Surg 1996;4:753-8.

9. Cronenwett JL, Birkmeyer JD, Nackman GB, et al. Costeffectiveness of carotid endarterectomy in asymptomatic patients. J Vasc Surg 1997;25:298-309; discussion 310-1.

10. Lee TT, Solomon NA, Heidenreich PA, et al. Cost effectiveness of screening for carotid stenosis in asymptomatic patients. Ann Int Med 1997;126:337-46.

11. Lavenson GS Jr. Carotid screening: preparing for the future. Vasc Ultrasound Today 1997;2:63-72.

12. Lavenson GS Jr. A new accurate, rapid and cost-effective protocol for stroke prevention screening. Cardiovasc Surg 1998;6:590-3.

13. Lavenson GS Jr. The carotid ultrasound report; practical considerations: Images, velocities and beyond. Vasc Ultrasound Today 1999;4:79-88.

14. Lavenson GS Jr. The Lav Scan and establishing a stroke prevention screening program. Vasc Ultrasound Today 2000;5:113-24.

15. Lavenson GS Jr. Ultrasound examination and management of carotid artery disease: Lessons learned. Vascular Ultrasound Today 2001;6:137-64.

16. Lavenson GS Jr. Stroke prevention screening: rationale, method and implementation. Vasc Ultrasound Today 2003;8:3-24.

17. Lavenson GS Jr, Pantera RL, Garza RM, et al. Development and implementation of a rapid, accurate, and cost-effective protocol for national stroke prevention screening. Am J Surg 2004;188:638-43.

18. Lavenson GS Jr. Ultrasound examination of the carotid arteries. Vasc Ultrasound Today 2004;9:163-92.

19. Lavenson GS Jr. The carotid artery ultrasound report: considerations in evaluation and management. J Vasc Ultrasound 2004;28:15-9.

20. Lavenson GS Jr. The American Vascular Association screening program: rationale, method, guidelines, and progress. J Vasc Ultrasound 2005;29:85-9.

21. Endarterectomy for asymptomatic carotid artery stenosis. Executive Committee for the Asymptomatic Carotid Atherosclerosis Study. JAMA 1995;273:1421-8.

22. Bulbulia R, Halliday A. The Asymptomatic Carotid Surgery Trial-2 (ACST-2): an ongoing randomised controlled trial comparing carotid endarterectomy with carotid artery stenting to prevent stroke. Health Technol Assess 2017;21:1-40.

23. Lavenson GS Jr. Why the U.S. Preventive Services Task Force recommendation against screening for asymptomatic carotid artery disease should be reconsidered. J Vasc Ultrasound 2012;36:26-30.

24. Pan XM, Saloner D, Reilly LM, et al. Assessment of carotid artery stenosis by ultrasonography, conventional angiography, and magnetic resonance angiography: correlation with ex vivo measurement of plaque stenosis. J Vasc Surg 1995;21:82-8; discussion 88-9.

25. Matsen SL, Chang DC, Perler BA. Trends in the inhospital stroke rate following Carotid endarterectomy in California and Maryland. J Vasc Surg 2006;44:488-95.

26. Jacobowitz GR, Rockman CB, Gagne PJ, et al. A model for predicting occult carotid artery stenosis: screening is justified in a selected population. J Vasc Surg 2003;38:705-9.

27. Rockman CB, Jacobowitz GR, Gagne PJ, et al. Focused 
screening for occult carotid artery disease patients with known heart disease are at high risk. J Vasc Surg 2004;39:44-51.

28. Paraskevas KI, Eckstein HH, Mikhailidis DP, et al. Rationale for screening selected patients for asymptomatic carotid artery stenosis. Curr Med Res Opin 2020;36:361-5.

29. Lavenson GS Jr. A three-step program to prevent strokes. Vasc Ultrasound Today 2007;12:3-49.

30. Mullenix PS, Martin MJ, Steele SR, et al. Rapid highvolume population screening for three major risk factors of future stroke: phase I results. Vasc Endovascular Surg 2006;40:177-87.

31. Lavenson GS Jr. Screening for arteriosclerotic vascular disease: a new program of scanning the femoral and carotid arteries and the aorta to prevent heart attacks, strokes, and ruptured aneurysms. Vasc Ultrasound Today 2009;14:23-38.

32. Lavenson GS Jr. Expansion of the SAAVE protocol with addition of ultrasound scans of the femoral and carotid arteries to prevent heart attacks and strokes. J Vasc Ultrasound 2011;35:92-6.

Cite this article as: Lavenson GS Jr, Andersen CA. The quick carotid scan for prevention of strokes due to carotid artery disease. Ann Transl Med 2021;9(14):1202. doi: 10.21037/atm20-7687 